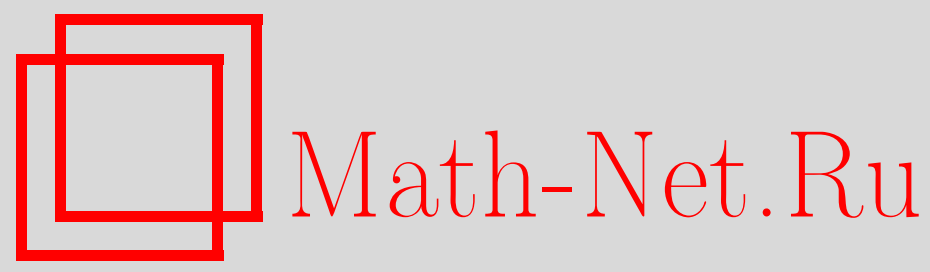

В. В. Гороховик, Е. А. Семенкова, Классификация полупространств по типам в бесконечномерных векторных пространствах, Матем. заметки, 1998, том 64, выпуск 2, 191198

DOI: https://doi.org/10.4213/mzm1385

Использование Общероссийского математического портала Math-Net.Ru подразумевает, что вы прочитали и согласны с пользовательским соглашением http://www.mathnet.ru/rus/agreement

Параметры загрузки:

IP: 34.229 .45 .116

26 апреля 2023 г., $17: 59: 28$

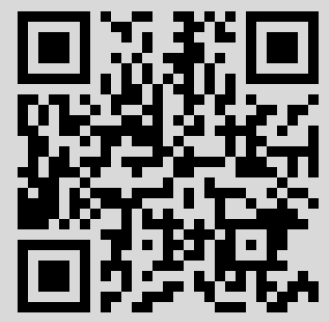


УДК $514.17+512.642$

\title{
КЛАССИФИКАЦИЯ ПОЛУПРОСТРАНСТВ ПО ТИПАМ В БЕСКОНЕЧНОМЕРНЫХ ВЕКТОРНЫХ ПРОСТРАНСТВАХ
}

\author{
В. В. Гороховик, Е. А. Семенкова
}

В работе показано, что каждое полупространство $C \subset X$ естественньм образом порождает на $X$ отношение полного предпорядка, относительно которого пара множеств $(X \backslash C, C)$ является сечением $X$. Отождествлением типа полупространства с типом порождаемого им сечения проведена классификация полупространств по типам, распространяющая на бесконечномерный случай классификацию полупространств в конечномерных векторных пространствах, данную Мартинец-Легазом и Зингером.

Библиография: 12 названий.

1. Введение. Пусть $X$ - вещественное векторное пространство.

Выпуклое множество $C$ из $X$ назьвается полупространством в $X$, если его дополнение $X \backslash C$ также является выпуклым множеством.

Очевидно, что если $C$ - полупространство, то $X \backslash C$ также является полупространством.

По-видимому, впервые полупространства рассматривались еще в работах Ш. Какутани [1] и Дж. Тьюки [2] в связи с общими теоремами об отделимости вьпуклых множеств. Позднее полупространства изучались в работах целого ряда авторов, в частности, в [3]-[9]. Недавно детальное исследование полупространств в конечномерных векторных пространствах было проведено в статье Х.-Э. Мартинец-Легаза и И. Зингеpa [6], одним из главных результатов которой является классификация полупространств в конечномерных векторных пространствах. Основана данная классификация на следующем аналитическом представлении конечномерных полупространств, доказательство можно найти в [6, теорема 2.1, утверждение (17)].

Теорема 1. Собственное подмнохсество С конечномерного векторного пространства $X$ является полупространством в $X$ тогда и только тогда, когда для некоторого натурального числа $m, 1 \leqslant m \leqslant \operatorname{dim} X$, существуют линейный оператор $A: X \rightarrow \mathbb{R}^{m}$ и вектор $\alpha=\left(\alpha_{1}, \ldots, \alpha_{m}\right) \in \mathbb{R}^{m}$ mакие, что $\operatorname{rank} A=m u$ либо

$$
C=\{x \in X \mid A x \leqslant L \alpha\}
$$

либо

$$
C=\left\{x \in X \mid A x<_{L} \alpha\right\} .
$$

Здесь " $\leqslant L " ~ u$ " ${ }_{L}$ " обозначают соответственно отношения лексикографического и строгого лексикографического порядка на $\mathbb{R}^{m}$.

Напомним (см., например, [7]), что векторы $u=\left(u_{1}, \ldots, u_{m}\right)$ и $v=\left(v_{1}, \ldots, v_{m}\right)$ из $\mathbb{R}^{m}$ удовлетворяют соотношению $u<_{L} v$ тогда и только тогда, когда $u \neq v$ и $u_{k}<v_{k}$, где 
$k=\min \left\{i \mid u_{i} \neq v_{i}, 1 \leqslant i \leqslant m\right\}$. Соотношение $u \leqslant L v$ эквивалентно тому, что либо $u=v$, либо $u<_{L} v$.

Линейньй оператор $A: X \rightarrow \mathbb{R}^{m}$ и вектор $\alpha \in \mathbb{R}^{m}$ в представлении собственного полупространства $C$, данном в теореме 1 , определяются неоднозначно. В то же время натуральное число $m, 1 \leqslant m \leqslant \operatorname{dim} X$, равное рангу линейного оператора $A$, а также тип представления (либо (1), либо (2)), которьй может быть задан в виде параметра $\tau$, принимающего значения из двухэлементного множества $\left\{\leqslant L,<_{L}\right\}$, являются инвариантами для каждого собственного полупространства $C$. Эти инварианты и положены Мартинец-Легазом и Зингером [6] в основу классификации конечномерньх полупространств. Согласно их классификации два собственных полупространства $C_{1}$ и $C_{2}$ принадлежат одному и тому же классу, если $m\left(C_{1}\right)=m\left(C_{2}\right)$ и $\tau\left(C_{1}\right)=\tau\left(C_{2}\right)$, т.е. если они имеют одинаковьй ранг и одинаковый тип. Как следует из теоремы 1 , в соответствии с этой классификацией в конечномерном векторном пространстве $X$, размерность которого равна $n$, существуют полупространства двух типов (типа “ $\leqslant L$ " и типа “ $<_{L}$ "), внутри же каждого типа полупространства разбиваются на $n$ различных классов в зависимости от их ранга. Таким образом, если $\operatorname{dim} X=n$, то в $X$ сушествует ровно $2 n$ различных классов полупространств.

Настоящая работа посвящена классификации полупространств в бесконечномерных векторных пространствах. Мы ограничимся лишь классификацией полупространств по “типу", не затрагивая их классификацию по "рангу". Наша классификация не связана с каким-либо аналитическим представлением полупространств, а основана на следующем внутреннем свойстве, присущем полупространствам. Мы устанавливаем (теорема 4), что любое собственное полупространство $C \subset X$ однозначно порождает на $X$ отношение полного предпорядка " $\leqslant c$ ", относительно которого пара множеств $(X \backslash C, C)$ является сечением пространства $X$. Тип данного сечения и отождествляется нами с типом полупространства $C$. Известно (см., например, [10]), что в любом упорядоченном множестве возможны только четыре типа сечений: скачок, два дедекиндовых (нижнее и верхнее) и щель. Мы показьваем, что

а) среди сечений пространства $X$, порожденных всевозможными его полупространствами, не сушествует скачков (теорема 5);

б) все сечения конечномерного векторного пространства $X$, порожденные его полупространствами, являются дедекиндовыми (теорема 6);

в) в любом бесконечномерном векторном пространстве $X$, кроме дедекиндовых полупространств, существуют полупространства, которые порождают сечения $X$, являющиеся щелями (теорема 7).

Таким образом, согласно нашей классификации в конечномерных векторных пространствах существуют полупространства двух различных типов, а в бесконечномерных векторных пространствах - трех типов. Ниже будет показано, что в конечномерных векторных пространствах наша классификация полупространств по их типам совпадает с соответствующей классификацией, данной в работе [6]. Перейдем к подробному изложению отмеченных выше результатов.

2. Некоторые свойства полупространств. Этот пункт имеет вспомогательньй характер. Установленные здесь свойства полупространств будут использованы нами при их классификации. Начнем с рассмотрения конических полупространств. Следуя [11], будем назьвать конус $K$ заостренным, если $0 \in K$, и незаостренным, если $0 \notin K$. 
Теорема 2. Заостренный выпуклый конус $K \subset X$ является полупространством тогда и только тогда, когда

$$
K \cup(-K)=X
$$

ДокАЗАТЕЛЬСТво. Необходимость. Пусть $K \subset X$-заостренный выпуклый конус, являюшийся полупространством. Тогда $M:=X \backslash K$-асимметричньй $(M \cap(-M)=\varnothing)$ выпукльй конус. Действительно, дополнение любого конуса есть конус. Поскольку $K-$ полупространство, то $M$ - выпуклый конус. Покажем, что $M$ является асимметричным. Предположим, что существует вектор $h \in M \cap(-M)$. В силу выпуклости $M$ получаем, что $0 \in M$. Это невозможно, поскольку $0 \in K$. Значит, $M \cap(-M)=\varnothing$. Заметим также, что равенство (3), которое необходимо доказать, эквивалентно равенству

$$
K \cup\left(-K^{>}\right)=X
$$

где $K^{>}=K \backslash(-K)$ - асимметричная часть конуса $K$. Так как $K \cap\left(-K^{>}\right)=\varnothing$, равенство (4) в свою очередь эквивалентно равенству $X \backslash K=-K^{>}$. Включение $-K^{>} \subset X \backslash K$ следует из определения асимметричной части. Рассмотрим вектор $h \in X \backslash K$. Поскольку $X \backslash K$ - асимметричньй выпукльй конус, то $-h \notin X \backslash K$ и, следовательно, $-h \in K$, причем $h \notin K$, т.е. $h \in-K^{>}$. Таким образом, $X \backslash K \subset-K^{>}$, что и требовалось показать.

Достаточность. Из равенства (3), а точнее из эквивалентного ему (4) следует, что $X \backslash K=-K^{>}$. Так как асимметричная часть любого вьпуклого конуса есть вьпуклый конус, то $X \backslash K$ является вьпуклым и, следовательно, $K$ есть полупространство.

СлЕДСТВИЕ. Асимметричный выпуклый конус $K \subset X$ является полупространством тогда и только тогда, когда множсество $X \backslash(K \cup(-K))$ является векторным подпространством.

Заметим, что вьпуклый конус $K$ асимметричен тогда и только тогда, когда он является незаостренным $(0 \notin K)$.

Пусть $C$ - произвольное множество из $X$. Рецессивным конусом множества $C$ назьвается (см. [11], [12]) множество $O^{+} C$, состоящее из векторов $h \in X$ таких, что $x+\gamma h \in C$ для всех $x \in C$ и всех действительных чисел $\gamma \geqslant 0$.

Нетрудно убедиться, что для любого множества $C \subset X$ его рецессивньй конус $O^{+} C$ является заостренным вьпуклым конусом.

ПРЕДЛОЖЕНИЕ. Для любого собственного подмножсества $C \subset X(C \neq X$, $C \neq \varnothing)$ справедливо равенство

$$
O^{+} C=-O^{+}(X \backslash C)
$$

ДокАЗАТЕЛЬСтво. Пусть $h \in O^{+} C$. Предположим, что $-h \notin O^{+}(X \backslash C)$. Тогда существуют $x \in X \backslash C$ и $\gamma>0$ такие, что $x-\gamma h \in C$. Так как $h \in O^{+} C$, то $x=(x-\gamma h)+\gamma h \in C$. Пришли к противоречию. Следовательно, $O^{+} C \subset-O^{+}(X \backslash C)$. Обратное включение доказьвается аналогично. 
Теорема 3. Собственное подмножество $C \subset X$ является полупространством тогда и только тогда, когда его рецессивный конус $\mathrm{O}^{+} \mathrm{C}$ также является полупространством.

ДокАЗАТЕльСтво. Необходимость. Поскольку рецессивньй конус $\mathrm{O}^{+} \mathrm{C}$ является заостренным выпукльм конусом, то в силу теоремы 2 достаточно показать, что $O^{+} C \cup$ $\left(-\mathrm{O}^{+} C\right)=X$. Предположим противное. Пусть существует $h \in X \backslash\left(\mathrm{O}^{+} C \cup\left(-\mathrm{O}^{+} C\right)\right)$. Тогда для любой точки $x \in C$ можно указать действительные числа $\gamma_{1}>0$ и $\gamma_{2}>0$ такие, что $x+\gamma_{1} h \in X \backslash C$ и $x-\gamma_{2} h \in X \backslash C$. Поскольку множество $X \backslash C$ выпукло, то весь отрезок, соединяющий эти точки, целиком принадлежит $X \backslash C$. Это невозможно, так как точка $x$ лежит на этом отрезке.

Достаточность. Предположим, что $\mathrm{O}^{+} \mathrm{C}$ является полупространством. Докажем, что множество $C$ вьпукло. Если множество $C$ не является вьпуклым, то существуют точки $x_{1}, x_{2} \in C$ и точка $z \in X \backslash C$, лежащая на отрезке, соединяющем точки $x_{1}, x_{2}$. Так как $O^{+} C$ - полупространство, хотя бы один из векторов $h_{1}:=x_{1}-z$ или $h_{2}:=x_{2}-z$ принадлежит $O^{+} C$. Действительно, включение $h_{1}, h_{2} \in-O^{>} C\left(O^{>} C-\right.$ асимметричная часть конуса $O^{+} C$ ) невозможно, поскольку $h_{1}=-\gamma h_{2}$, где $\gamma>0$. Пусть для определенности $h_{1} \in O^{+} C$. Тогда в силу теоремы $2-h_{1} \in O^{+}(X \backslash C)$, значит $x_{1}=z+\left(-h_{1}\right) \in X \backslash C$. Пришли к противоречию. Следовательно, $C$ является вьпуклым. Аналогично доказьвается вьпуклость $X \backslash C$.

3. Классификация полупространств по типам. Напомним некоторые понятия из теории упорядоченных множеств (см., например, [10]).

Множество $X$ называется упорядоченным, если на нем задано отношение полного предпорядка, т.е. рефлексивное и транзитивное бинарное отношение " $\leqslant$ ", удовлетворяюшее условию полноты: для любых $x, y \in X$ вьполняется хотя бы одно из соотношений $x \leqslant y$ или $y \leqslant x$.

Сечением упорядоченного множества $X$ назьвается его разбиение на два непересекающихся подмножества $A$ и $B$ такое, что для любых элементов $x \in A, y \in B$ выполняется $x<y$, где $x<y$ означает $x \leqslant y, y \nless x$. Множество $A$ называется нижним классом, а множество $B-$ верхним классом сечения. Сечения, у которых $A \neq \varnothing$ и $B \neq \varnothing$, называются собственными.

Возможны следующие типы собственных сечений.

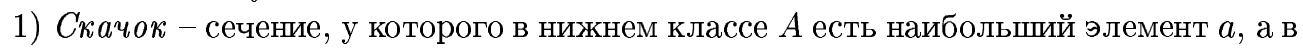
верхнем классе $B$ есть наименьший элемент $b$ (очевидно, что в этом случае не существует $x \in X$ такого, что $a<x<b)$.

2) Верхнее дедекиндово сечение - сечение, у которого в нижнем классе $A$ нет наибольшего элемента, а в верхнем классе $B$ есть наименьший элемент.

3) Нижнее дедекиндово сечение - сечение, у которого в нижнем классе $A$ есть наибольший элемент, а в верхнем классе $B$ нет наименьшего элемента.

4) Щель - сечение, у которого в нижнем классе $A$ нет наибольшего элемента, а в верхнем классе $B$ нет наименьшего элемента.

Пусть $C$ - полупространство в вещественном векторном пространстве $X$ и $O^{+} C-$ рецессивньй конус $C$. Определим на $X$ отношение полного предпорядка “ $\leqslant c$ ", полагая

$$
x \leqslant c y \Longleftrightarrow y-x \in O^{+} C
$$

(транзитивность и рефлексивность отношения " $\leqslant c$ " следуют из вьпуклости и заостренности конуса $O^{+} C$, а полнота - из того, что $O^{+} C$ является полупространством). Таким 
образом, отношение " $\leqslant c$ " превращает вещественное векторное пространство $X$ в упорядоченное множество.

ТЕОРема 4. Для любого полупространства $C$ в вещественном векторном пространстве $X$ пара $(X \backslash C, C)$ является сечением пространства $X$, упорядоченного отношением полного предпорядка " $\leqslant c "$ ".

ДокаЗАТЕЛЬСтво. Если для некоторых $x \in C$ и $y \in X \backslash C$ выполняется отношение $x \leqslant c$, то, поскольку оно эквивалентно включению $y-x \in O^{+} C$, имеем $y=$ $x+(y-x) \in C$, что невозможно. Значит, $y<_{c} x$ для всех $x \in C, y \in X \backslash C$.

Теорема 5. Среди сечений вещественного векторного пространства $X$, порожденных всевозмохными его полупространствами, не существует скачков.

ДокаЗАТЕЛЬСтво. Предположим противное. Пусть $C$-полупространство в $X$ такое, что порожденное им сечение $(X \backslash C, C)$ есть скачок. Пусть $a$ - наибольший (относительно “ $\leqslant c$ ") элемент в $X \backslash C$, а $b$-наименьший элемент в $C$. Из определения сечения следует, что $a<_{c} b$, т.е. $b-a \in O^{+} C$, причем $a-b \notin O^{+} C$. Рассмотрим произвольный вектор $c=a+\gamma(b-a)$, где $\gamma$ - некоторое фиксированное число из $(0,1)$. Так как $c-a=\gamma(b-a)$, причем $\gamma>0$, то $c-a \in O^{+} C, a-c \notin O^{+} C$, т.е. $a<_{c} c$. Следовательно, $c \notin X \backslash C$, поскольку $a$ - наибольший элемент в $X \backslash C$. С другой стороны, так как $b-c=(1-\gamma)(b-a)$ и $\gamma \in(0,1)$, то $b-c \in O^{+} C, c-b \notin O^{+} C$, т.е. $c<_{c} b$. Следовательно, $c \notin C$, ибо $b$ есть наименьший элемент в $C$. Таким образом, $c \notin X \backslash C$ и $c \notin C$, что невозможно.

Приведем примеры полупространств, порождающих дедекиндовы сечения.

ПримеР 1. Любое полупространство $C$ в множестве действительных чисел $\mathbb{R}$ порождает на $\mathbb{R}$ отношение порядка " $\leqslant c$ ", которое совпадает либо с естественньп порядком, либо с противоположньм ему порядком на $\mathbb{R}$. Как известно (см., например, [10]), любое сечение множества действительных чисел относительно естественного (значит, и противоположного ему) порядка является дедекиндовым. Заметим, что нижний и верхний классы любого сечения множества действительных чисел относительно естественного порядка являются полупространствами в $\mathbb{R}$.

ПримеР 2. Пусть $X=\mathbb{R}^{2}$. Нетрудно проверить, что множество

$$
C:=\left\{\left(x_{1}, x_{2}\right) \in \mathbb{R}^{2} \mid x_{1}>\alpha_{1}\right\} \cup\left\{\left(x_{1}, x_{2}\right) \in \mathbb{R}^{2} \mid x_{1}=\alpha_{1}, x_{2}>\alpha_{2}\right\},
$$

где $\alpha_{1}, \alpha_{2}$ - заданные действительные числа, является полупространством. Соответствующее ему отношение порядка " $\leqslant c$ " есть отношение лексикографического порядка на $\mathbb{R}^{2}:\left(x_{1}, x_{2}\right) \leqslant c\left(y_{1}, y_{2}\right)$ тогда и только тогда, когда либо $x_{1}<y_{1}$, либо $x_{1}=y_{1}$, $x_{2} \leqslant y_{2}$. Нижний класс $X \backslash C$ сечения, порожденного $C$, обладает единственньг наибольшим элементом $\alpha=\left(\alpha_{1}, \alpha_{2}\right)$ и, следовательно, сечение $(X \backslash C, C)$ является нижним дедекиндовым.

Полупространство, порождающее верхнее дедекиндово сечение пространства $X$, будем назьвать заостренным дедекиндовым, а полупространство, порождающее нижнее дедекиндово сечение, - незаостренным дедекиндовым.

Из равенства (5) следует, что если $C$ является заостренным (незаостренным) дедекиндовым полупространством, то его дополнение $X \backslash C$ есть незаостренное (заостренное) дедекиндово полупространство. 
ТЕОРема 6. Любое собственное полупространство конечномерного векторного пространства является дедекиндовым (заостренным или незаостренным).

ДокАЗАТЕЛЬСТво. В силу теоремы 1 любое собственное полупространство $C$ конечномерного векторного пространства $X$ может быть представлено либо в виде (1), либо в виде $(2)$. Пользуясь линейностью оператора $A$ и свойствами отношений “ $\leqslant L$ ” и “ $<_{L}$ ", можно показать, что (независимо от того, имеет ли полупространство $C$ вид (1) или вид (2)) для рецессивного конуса $O^{+} C$ справедливо представление $O^{+} C=\{x \in X \mid$ $\left.A x \leqslant_{L} 0\right\}$. Так как линейньй оператор $A$ невырожден, аффинное подпространство $M:=\{x \in X \mid a x=\alpha\}$ непусто и либо $M \subset C$, если $C$ имеет вид (1), либо $M \subset X \backslash C$, если $C$ имеет вид (2). Нетрудно убедиться, что в случае $M \subset C$ любая точка $x \in M$ является наименьшей в $C$ относительно “ $\leqslant c$ " и, следовательно, $C$ является заостренным дедекиндовым полупространством. Если же $M \subset X \backslash C$, то любая точка $x \in M$ является наибольшей в $X \backslash C$ относительно " $\leqslant c$ " и в этом случае $C$ является незаостренным дедекиндовым полупространством.

ЗАмЕчАнИЕ. Из доказательства теоремы 6 следует, что заостренные дедекиндовы полупространства конечномерного векторного пространства, и только они, имеют в классификации Мартинец-Легаза и Зингера тип " $\leqslant L$ ", а незаостренные дедекиндовы полупространства, и только они, имеют тип " $<_{L}$ ".

ТЕОремА 7. В любом бесконечномерном векторном пространстве $X$ существуют полупространства, которые порождают сечения $X$, являющиеся щелями.

ДокАЗАтЕльство. Зафиксируем в $X$ произвольный базис Гамеля $H=\left\{e_{j}\right\}_{j \in J}$. Так как $H$ бесконечно, выберем в нем счетное подмножество $E$ и занумеруем его элементы натуральными числами, тем самьм упорядочив $E$ подобно натуральному ряду $\mathbb{N}$. Для каждого вектора $x \in X$ через $\left\{x_{i}\right\}_{i=1}^{\infty}$ будем обозначать те его координаты в базисе $H$, которые соответствуют базисньм векторам из $E$. Поскольку лишь конечное число координат вектора $x$ в базисе $H$ может быть отлично от 0 , сумма $\sum_{i=n}^{\infty} x_{i}$ имеет смысл для каждого вектора $x \in X$ и каждого натурального числа $n$.

Определим в $X$ последовательность подмножеств

$$
C_{n}^{+}:=\left\{x \in X \mid \sum_{i=n}^{\infty} x_{i}>1, \sum_{i=s}^{\infty} x_{i}=1, s<n\right\}, \quad n=1,2, \ldots
$$

Непосредственно проверяется, что для каждого $n=1,2, \ldots$ подмножество $C_{n}^{+}$является выпукльгм. Кроме того, если $m<n$, то для любого $x \in C_{m}^{+}$и любого $y \in C_{n}^{+}$включение $\gamma x+(1-\gamma) y \in C_{m}^{+}$имеет место для всех $\gamma \in(0,1]$. Действительно, для любого $y \in C_{n}^{+}$ выполняются равенства $\sum_{i=s}^{\infty} y_{i}=1$ при всех $s=1, \ldots, m$. Для любого же $x \in C_{m}^{+}$ имеем $\sum_{i=s}^{\infty} x_{i}=1$ при всех $s=1, \ldots, m-1$ и $\sum_{i=m}^{\infty} x_{i}>1$. Следовательно, для суммы векторов $\gamma x+(1-\gamma) y$ при любом $\gamma \in(0,1]$ имеем

$$
\sum_{i=s}^{\infty}\left(\gamma x_{i}+(1-\gamma) y_{i}\right)=1, \quad s=1, \ldots, m-1, \quad \sum_{i=m}^{\infty}\left(\gamma x_{i}+(1-\gamma) y_{i}\right)>1
$$

Значит, $\gamma x+(1-\gamma) y \in C_{m}^{+}$для всех $\gamma \in(0,1]$. 
Рассмотрим теперь множество $C:=\bigcup_{n=1}^{\infty} C_{n}^{+}$. В силу доказанных вьше свойств подмножеств $C_{n}^{+}, n=1,2 \ldots$, множество $C$ выпукло. Покажем, что $C$ является полупространством в $X$. С этой целью введем последовательность подмножеств

$$
C_{n}^{-}:=\left\{x \in X \mid \sum_{i=n}^{\infty} x_{i}<1, \sum_{i=s}^{\infty} x_{i}=1, s<n\right\}, \quad n=1,2, \ldots,
$$

и заметим, что $X \backslash C=\bigcup_{n=1}^{\infty} C_{n}^{-}$. Действительно, пусть

$$
I(x):=\left\{n \in \mathbb{N} \mid \sum_{i=n}^{\infty} x_{i} \neq 1\right\} .
$$

Докажем, что $I(x) \neq \varnothing$ для любого $x \in X$. Условие $I(x)=\varnothing$ эквивалентно тому, что $\sum_{i=n}^{\infty} x_{i}=1$ для всех $n \in \mathbb{N}$. Однако, если $x_{m}$ - ненулевая координата вектора $x$ с наибольшим индексом $m$, то $\sum_{i=n}^{\infty} x_{i}=0$ для всех $n>m$. Следовательно, никакой вектор $x \in X$ не может удовлетворять равенствам $\sum_{i=n}^{\infty} x_{i}=1$ для всех $n \in \mathbb{N}$. Пусть $x \in X \backslash C$ и $k=k(x)$ - наименьший элемент в $I(x)$. Тогда $\sum_{i=n}^{\infty} x_{i}=1$ для всех $n<k$ и $\sum_{i=k}^{\infty} x_{i}<1$, поскольку $x \notin C$ и $k \in I(x)$. Значит, $x \in C_{k}^{-}$и, следовательно, $X \backslash C \subset \bigcup_{n=1}^{\infty} C_{n}^{-}$. Обратное включение очевидно. С использованием равенства $X \backslash C=\bigcup_{n=1}^{\infty} C_{n}^{-}$вьпуклость множества $X \backslash C$ доказьвается аналогично вьпуклости $C$. Таким образом, оба множества $C$ и $X \backslash C$ являются выпуклыми и, следовательно, $C$ есть полупространство в $X$.

Докажем теперь, что сечение $X$, которое порождается полупространством $C$, является щелью. Для этого построим рецессивньй конус множества $C$. Введем последовательность подмножеств

$$
M_{n}:=\left\{x \in X \mid \sum_{i=n}^{\infty} x_{i}>0, \sum_{i=s}^{\infty} x_{i}=0, s<n\right\}, \quad n=1,2, \ldots,
$$

и множество

$$
M_{0}:=\left\{x \in X \mid \sum_{i=n}^{\infty} x_{i}=0, n=1,2, \ldots\right\} .
$$

Заметим, что $M_{0} \neq \varnothing$, так как $0 \in M_{0}$.

Покажем, что

$$
O^{+} C=\bigcup_{n=0}^{\infty} M_{n}
$$

Пусть $h \in M_{n}$ и $x \in C_{k}^{+}$, где $n$ и $k$ - некоторые фиксированные натуральные числа. Если $k \leqslant n$, то $x+\gamma h \in C_{k}^{+}$для всех $\gamma \geqslant 0$. Если же $n<k$, то $x+\gamma h \in C_{n}^{+}$для всех $\gamma>0$. В любом случае имеем $x+\gamma h \in C$ для всех $x \in C, h \in \bigcup_{n=0}^{\infty} M_{n}, \gamma \geqslant 0$. Значит, $\bigcup_{n=0}^{\infty} M_{n} \subset O^{+} C$. Для доказательства обратного включения заметим, что для любого вектора $h \notin \bigcup_{n=0}^{\infty} M_{n}$ существует номер $n \in \mathbb{N}$, при котором $-h \in M_{n}$. Но в этом случае для любого $x \in \bigcup_{k=n+1}^{\infty} C_{k}^{+} \subset C$ имеем $x+\gamma h \in C_{n}^{-}$для всех $\gamma>0$ и, следовательно, $h \notin O^{+} C$. Значит, $X \backslash \bigcup_{n=0}^{\infty} M_{n} \subset X \backslash O^{+} C$, что влечет $O^{+} C \subset \bigcup_{n=0}^{\infty} M_{n}$. Таким образом, равенство (6) доказано.

Покажем теперь, что в $C$ нет $\leqslant c$-наименьшего элемента. С этой целью заметим, что при $m<k$ и только в этом случае отношение $y<_{c} x$ имеет место для всех $y \in C_{k}^{+}$и 
всех $x \in C_{m}^{+}$. Действительно, для любого $y \in C_{k}^{+}$имеем $\sum_{i=n}^{\infty} y_{i}=1$ при всех $n \leqslant m$, а для любого $x \in C_{m}^{+}$имеем $\sum_{i=n}^{\infty} x_{i}=1$ при всех $n<m$ и $\sum_{i=m}^{\infty} x_{i}>1$. Значит, вектор $h:=x-y$ удовлетворяет условиям $\sum_{i=n}^{\infty} h_{i}=0$ для всех $n<m$ и $\sum_{i=m}^{\infty} h_{i}>0$. Следовательно, $h \in M_{m} \subset O^{+} C$, причем $-h \notin O^{+} C$. Таким образом, $y \leqslant c x$, но $x \nless c y$, т.е. $y<_{c} x$.

Из этого замечания следует, что для любого вектора $x \in C$ существует $y \in C$, удовлетворяющий соотношению $y<_{c} x$. На самом деле, поскольку $x \in C$, то $x \in C_{m}^{+}$при некотором $m \in \mathbb{N}$, и, следовательно, любой вектор $y \in C_{k}^{+} \subset C$, где $k$ - произвольное натуральное число такое, что $m<k$, удовлетворяет отношению $y<_{c} x$.

Аналогично доказьвается, что в $X \backslash C$ нет $\leqslant c$-наибольшего элемента. Таким образом, построенное множество $C$ является полупространством, которое порождает сечение $X$, являющееся щелью.

Полупространство, которое порождает сечение векторного пространства, являющееся щелью, будем назьвать для краткости полупространство-щель.

Итак, всеполупространства любого бесконечномерного векторного пространства делятся по типам порождаемых ими сечений векторного пространства на три непустых непересекающихся класса:

1) класс заостренных дедекиндовых полупространств, которые порождают верхние дедекиндовы сечения;

2) класс незаостренных дедекиндовых полупространств, которые порождают нижние дедекиндовы сечения;

3) класс полупространств-щелей, которые порождают сечения, являющиеся щелями.

В любом конечномерном векторном пространствекласс полупространств-щелей пуст, и наша классификация совпадает с классификацией Мартинец-Легаза и Зингера [6], при этом верхним дедекиндовым полупространствам соответствуют полупространства типа " $\leqslant L$ ", а нижним дедекиндовым полупространствам - полупространства типа " $<L$ ".

\section{СПИСОК ЦИТИРОВАННОЙ ЛИТЕРАТУРЫ}

[1] Kakutani S. Ein Beweis des Satzes von M. Eidelheit über konvexe Mengen // Proc. Imp. Acad. Japan. 1938. V. 14. P. 93-94.

[2] Tukey J.W. Some notes on the separation of convex sets // Portugal. Math. 1942. V. 3. P. 95-102.

[3] Hammer P. C. Maximal convex sets // Duke Math. J. 1955. V. 22. P. 103-106.

[4] Klee V. L. The structure of semispaces // Math. Scand. 1956. V. 4. P. 54-64.

[5] Martinez-Legaz J.-E. Exact quasiconvex conjugation // Z. Oper. Res. 1983. V. 27. P. 257-266.

[6] Martinez-Legaz J.-E., Singer I. The structure of semispaces in $\mathbb{R}^{n} / /$ Linear Algebra Appl. 1988. V. 110. P. 117-179.

[7] Martinez-Legaz J.-E., Singer I. Lexicographical order, lexicographical index, and linear operators // Linear Algebra Appl. 1990. V. 128. P. 65-95.

[8] Гороховик В. В. Выпуклые задачи векторной оптимизации. Минск: Наука и техника, 1990.

[9] Lassak M. Convex half-spaces // Fund. Math. 1984. V. 120. P. 7-13.

[10] Александров П. С. Введение в теорию множеств и общую топологию. М.: Наука, 1977.

[11] Рокафеллар Р. Т. Выпуклый анализа. М.: Мир, 1973.

[12] Luc D.T. Recession cones and the domination property in vector optimization // Math. Programming. 1990. V. 49. №1. P. 113-122. 